\title{
What Information Propagates among the Public when an Initial Coin Offering (ICO) is Initiated? A theory-driven approach
}

\author{
Soo Il Shin \\ Missouri State University \\ sooshin@missouristate.edu \\ Dianne J. Hall \\ Auburn University \\ halldia@auburn.edu
}

\author{
Joo Baek Kim \\ University of Tampa \\ jkim@ut.edu \\ Teresa Lang \\ Auburn University - Montgomery \\ tlang@aum.edu
}

\begin{abstract}
Since the popularity of blockchain-based cryptocurrency investments has increased among the public, people have directly purchased cryptocurrency through the cryptocurrency market or joined initial coin offering (ICO) projects. This research explores what informational cues are captured before, during, and after ICO projects that can be considered as signals and a fulfillment of information asymmetry. We adopted two theoretical underpinnings to achieve our research goal - agency and signaling theory. Using information from Twitter, we selected the best-performing ICO project based on the highest return on investment (ROI). Then, we extracted 5,085 tweets related to the selected ICO project. Tweets are categorized by pre-ICO, during and post-ICO, by topic, and dispersion. Analyzing the tweets, we found multiple categories of informational cues for each ICO project. Implications and limitations are discussed.
\end{abstract}

\section{Introduction}

Cryptocurrency is a digital currency or money that uses cryptographic technology. The concept of digital currency is not a new and is gaining acceptance across society. As a result, investors are focusing significant attention toward bitcoin investments.

Cryptocurrency uses open-source algorithms, allowing any party to issue cryptocurrencies to members both inside and outside the network without a legitimate authorizing party. Currently, the most well-known cryptocurrencies are Bitcoin and Ethereum. Bitcoin uses a proof-of-work (PoW) consensus protocol. This protocol employs block miners to verify transactions. Each block miner is awarded bitcoins if he/she successfully verifies transactions, so they can link a new block to the existing blockchain. However, the total number of bitcoins is already set to 21 million, and the reward for mining is a half of a bitcoin for every 210,000 blocks verified [7]. It is well known that the mining difficulty tends to increase if the number of bitcoins is increasing.

Bitcoin is now tradable over the network electronically through exchange markets. The number of exchange markets has reached more than 10,645 [1] and is expected to continue increasing [17]. Along with Bitcoin and Ethereum, Ripple, Bitcoin Cash, and EOS ranked among the first five cryptocurrencies in terms of market capitalization [1]. Trading markets have rapidly increased; causing price volatility to become an issue. For instance, the price of bitcoin started fluctuating drastically in 2017, with the price peaking on December 15,2017 at $\$ 17,586.80$ per coin. - compared to $\$ 972.95$ per coin on January 1, 2017. Other coins also have high volatility during this period. Ether, the second most popular cryptocurrency, increased in price from $\$ 15$ in March 2017 to \$1,377 in January 2018, and continues to have significant price fluctuations. As result, many individuals have been attracted to cryptocurrency investing to earn profits similar to, or exceeding, traditional stock and bond investments. 
In addition to investing and/or transaction validation, individuals can earn cryptocurrency by participating in initial coin offering (ICO). ICO is a means of fundraising through which organizations or new projects sell digital tokens in exchange for bitcoins or other similar cryptocurrencies. ICO is gaining popularity among startup companies because of the lack of government regulation. An initial public offering (IPO) sells shares of an organization to the public, while ICOs sell their own crypto-tokens to those who are willing to support startup companies. Thus, to meet the startup company's initial target capital, the company should prepare a project plan regarding what they will achieve, funding required to conduct the project, how long ICO campaigns will last, and so on. Unlike an IPO, which is a means of investment, ICOs are regarded as donations or cloud sales, because a distributed cryptotoken is not a financial asset but rather a digital good not governed by regulatory financial agencies (e.g., SEC).

Such token holders may benefit because the tokens are exchangeable, so the initial buyers can resell their shares if they believe the value of the token(s) increased. From the standpoint of startups or token-issuers, initiating ICO is one of the easiest ways to raise funds for their projects. Additionally, ICO issuers can enjoy the benefit of decentralized business options for their project. From an ICO buyer's standpoint, they can achieve monetary gain if the project runs well or the token prices increase in the exchange market. However, participants must bear the risk of default or fraud since there is no regulating party. Although such concerns have existed since 2013, when the first token appeared, the huge success of Ripple and Ethereum, make ICOs very popular. Particularly, in 2016, roughly $\$ 256$ million was raised by startups. Although there are success stories of buyers earning profits through CIO transactions, buyers should be cautious because any loss of funds is not recoverable under any governing regulation.

Given the current popularity of cryptocurrencies among the public, and the interest in the different ways to invest in cryptocurrency, our research focuses on ICO projects issuing cryptocurrency tokens which are freely exchangeable for legitimate cryptocurrency (e.g., bitcoins, ETS or others). However, since ICO projects are not a conventional method for raising startup capital, potential investors have limited access to sufficient information to make a decision about ICO participation. Particularly, because the concept of blockchain is still gaining momentum, limited public information may hinder the success of ICO projects.

Our research endeavors to capture either the informational cues generated by an ICO project officially or any potential public investors. Therefore, the purpose of this research is to extract informational cues from ICO projects and potential public investors in order to identify what information is considered important among them. To extract informational cues related with ICO projects, our research examined social media feeds-particularly tweets from Twitter users. Almost all ICO projects open their own Twitter account to spread the newest or updated project information to the public. Also, many people follow their official Twitter account to learn or share helpful information. Thus, we raise the following research questions:

RQ1: what kind of information is disseminated to the public generated by any parties including ICO projects or public Twitter users?

RQ2: what are the most important informational cues before, during, and after an ICO project?

We adopted two theoretical underpinnings: 1) agency theory and 2) signaling theory. Agency theory [5] explains why information asymmetry occurs between information recipients and senders, and how they pursue their own interest. Signaling theory [18], another backbone of the current research, is used to explain the flow of information from one party to another when the information is asymmetric between those parties [22]. Supported by these two theories, we are particularly interested in indicators just before the occurrence of unusual transactions, and what kind of signals may be detected. For a research method, we adopted a text mining technique to extract keywords from social media outlets.

\section{Literature and technology review}

\subsection{Blockchain technology and cryptocurrency}


To understand how to obtain cryptocurrency, it is important to understand its undergirding technology called Blockchain. Learning Blockchain is important because cryptocurrency is one of the most rewarding ways to maintain Blockchain technology and verify all transactions conducted over Blockchain. Blockchain is an emerging technology that is changing the concept of transactions involved in intermediary parties between entities and is leading a new world of securing information. The seminal concept of Blockchain technology was raised by an unknown person or party named Satoshi Nakamoto in 2008 [13]. Satoshi Nakamoto suggested a distributed ledger that records all transaction histories, and the ledger is shared with all parties simultaneously. All transactions should go through a validation process called a decentralized consensus algorithm, and valid transactions are stored in a block. The chain of blocks eventually contains all historical transactions and any parties are able to download a copy of Blockchains. Each block can contain the limited number of transactions so that once the block is full then another block starts filling in following transactions.

Those blocks linked together chronologically through mathematical processes called Blockchain mining under consensus protocol. To achieve anonymous consensus for all transactions on the distributed ledger system, Blockchain technology has developed its own consensus algorithms conducted by all nodes. Popular consensus mechanisms include Proof-of-Work (PoW), Proof-of-Stake (PoS), and their variations (e.g., distributed PoS or delayed PoW). Proof-of-Work (PoW) is one of the consensus protocols that has been widely used since Satoshi Nakamoto created it. PoW requires people in the Blockchain node, called miners, who solve cryptographic puzzles voluntarily to make a chain of blocks. For instance, technically, those miners should solve a mathematical problem rooted from a hash function. The problem can only be answered by brute force guessing, meaning that nonce-appended hash is answered when a miner tries too many combinations of a message and random integer values. Such unidirectional guessing processes stem from the irreversibility of a hash function. Once an individual solves the function, the block is added in an existing chain if all other parties verify the validity of the answer. Then, such individuals also receive a reward in the form of cryptocurrency. PoW requires a huge amount of computing power and electronic resources if the number of nonce increases and the speed of adding blocks slows, or if the level of difficulty increases. Therefore, earning cryptocurrency by mining only becomes more difficult over time. To remedy such mathematical challenges, other consensus protocols also were developed. For example, Proof-of-Stake (PoS) is another well-known consensus protocol. While PoW requires the solving of the hash function by a miner, PoS selects a limited number of stakeholders in the transaction system and thus let them create blocks depending on their stake in the network. Those stakeholders are not rewarded in cryptocurrency, but they do take transaction fees.

Benefitting from such Blockchain structure, stored transaction data sustains its integrity and security [11]. Additionally, immutability of Blockchain makes it nearly impossible to forge transactions stored in blocks, thereby establishing mutual trust among transacting parties without the "middle man" or third party [20]. Since Blockchain technology has publicly transparent transaction systems, all transacting parties have accessibility to blocks to read; however, public accessibility is not always desirable for certain groups of a community or organizations [8]. Thus, the need for private-based Blockchain becomes apparent, as it allows only a limited number of entities to access transaction records and share within those participants [8]. The benefit of private Blockchain is securing information or transactions among predetermined entities. Another type of Blockchain is called a permissioned Blockchain [15]. Under the permissions of the Blockchain system, pre-selected entities determine the validity of transactions; thus, consensus is made quickly when compared to other Blockchain systems.

Because of participating mining activities that require significant time and monetary resources, for earning cryptocurrency, many cryptocurrency markets are flourishing. The number of cryptocurrency by mining is scarce, buying and selling them is competitive. Therefore, without joining the mining process, the general public can earn cryptocurrency by purchasing cryptocurrency in the market or joining ICO projects 
that distribute cryptocurrency tokens that are exchangeable to cryptocurrency.

\section{Theoretical Background}

\subsection{Agency theory}

Agency theory [5] is a theoretical underpinning that explains the relationship between a principal and agent. In the relationships between principals and agents, those two parties form a physical or mutual contract through which an agent completes work as a delegate of the principal. The theory also acknowledges information asymmetry or incomplete information between two parties. From a principal standpoint, given incomplete information regarding an agent, a principal can minimize risk associated with the agent's behavior by purchasing information about the agent or rewarding him or her on performance [18]. Because a principal and agent pursue a goal differently - even when the agent is a surrogate of the principal's work - agency costs such as monitoring cost, bonding cost and residual expenses, are avoidable [6]. Under the basic notion of agency theory, information systems (IS) researchers adopted the theory in relation to the buyer and seller relationship in the contexts of e-commerce or IT outsourcing, etc. (e.g., [14]). For instance, in the e-commerce context, sellers (agents) are responsible for the product delivery to the buyers (principals), and the sellers tend to have more product information than buyers do [14]. Because of the transacting conditions over the network in ecommerce, usually the buyer has only limited access to information regarding products, particularly when compared to the information available to the seller. Thus, such information imbalances cause information asymmetry between the seller and the buyer, introducing uncertainty regarding transactions between parties [9]. To mitigate such uncertainty or risk, the buyer-as a principal-incurs agency costs, such as monitoring, bonding, and residual costs. For example, there is no way to physically evaluate products inperson during e-commerce transactions, resulting in implicit monitoring costs. Any delivery delay or partial/total loss of products on the delivery might cause bonding and residual cost, respectively.

Our research focuses on mitigating agency costs caused by information asymmetry when investing in cryptocurrency via the ICO market. From the standpoint of a prospective investor or other person interested in cryptocurrency, information asymmetry directly effects profit and loss. In particular, it becomes a serious consideration when market volatility is extreme. Contrary to a conventional shopping environment, where buyers may use publicly available online review features to evaluate affordability, manufacturer or brand, quality, and product history to reduce uncertainty and the risk of monetary loss. The people who are interested in ICO markets are more likely to encounter a lack of such open information; thereby, increasing their level of uncertainty. Our study puts the person interested in an ICO project in the position of principal, and the ICO project as an agent. We then examine how they mitigate information asymmetry, by looking for informational cues as evidence of their efforts to compensate the imbalance in shared information between parties. All sharing or generating information before, during, or after the ICO project can be deemed an effort to reduce agency cost including monitoring, bonding, and residual cost.

\subsection{Signaling theory}

Signaling theory [18] is a popular theoretical backbone in many academic disciplines, such as finance, information systems (IS), and marketing. It is commonly used to evaluate the flow of information from one party to another when the informational conditions of the respective parties are asymmetrical [22]. Rao, Lu and Ruekert [16] define a signal as "an action that the seller can take to convey information credibly about unobservable product quality to the buyer" (p. 259), which plays an important role in alleviating the asymmetry of information. Kirmani and Rao [10] addressed four conditions (pre-purchase information scarcity, post-purchase information clarity, payoff transparency, and bond vulnerability) of signal transmissions. Whereas pre-purchase information scarcity refers to the absence of available and accessible information about product quality, post-purchase information clarity happens "when a consumer can readily assess the quality of a product immediately after purchase or use" [22, p. 375]. In the context of the buyer and seller relationship, high signal credibility exists "when consumers believe that the seller made a significant investment by sending a signal and the investment is at risk if a false signal is sent" [22, p. 376]. 
Sending false signals can result in sellers incurring financial costs and can hurt the organization's reputation or image, wealth, and investments. Low signal credibility reduces buyers' confidence in the quality of products, which has a greater influence on repetitive sales firms versus fly-by-night firms [2, 12]. High quality sellers usually inform buyers of the true quality of products and maintain their high credibility by avoiding false signaling. Sending high quality signals evidently requires significant extrinsic (e.g., additional financial costs, more inventory) and intrinsic (e.g., hiring additional human resources) investments on the part of the company.

The current study examines any signals that can be regarded as informational cues, such as, delivering information, and the sender's capability of providing credible information to a recipient [14]. In particular, our research examines the messages generated in terms of what makes ICO projects credible before, during, and after an ICO project. While highly credible information and its dissemination to the public is important, sending false or unreliable information that results in financial loss hurts the ICO project initiator. Therefore, high quality ICO initiators are more likely to share more trustworthy, reliable information about projects and producing a high quality signal.

\section{Research Method}

\subsection{ICO project overview}

For our research artifacts, we selected Stratis Company (www.stratisplatform.com) which provides a Blockchain-as-a-Service (BaaS) platform and offers blockchain technology to the financial sector. Stratis offers scalable services and applications, a customized private blockchain, a simple blockchain developmentprocessing algorithm, and a $\mathrm{C \#}$ built on full node platform. Stratis platform enables a company to easily incorporate existing features from other blockchains such as Ethereum [21].

In 2016, to fund the development of the Stratis platform, Stratis initiated an ICO to the public from June 21 to July 26 [4]. The company raised a total of $\$ 610,908$, collected from 915 bitcoins during the ICO period with an initial token price of $\$ 0.0073$ by 509 investors [19]. For the first five ICO days, investors received a $20 \%$ bonus and this bonus reached zero in the last 11 days [4]. After ICO, 98 million tokens were distributed to ICO investors $(85.7 \%)$ and the core team $(14.3 \%)$. At the time of the data analysis, return on investment (ROI) recorded 79,783.90\% which is the highest ROIs among ICO projects currently in running and the token price reached \$5.86 as of May 17, 2018 .

\subsection{Data collection}

Data were collected from the tweets regarding Stratis blockchain platform posted on Twitter.com between May 22and August 26, 2016. The period was chosen because the Stratis blockchain held the initial coin offering between June 21 and July 26, 2016. We deemed that a month before- and after-ICO give substantial amounts of tweets to find meaningful signals from the principal and the agents of ICO event. To do so, we extracted tweets that included any of following terms/hashtags: "Stratis", "\#stratis", and "\$Strat" using the twitter extraction tool named GetOldTweets from Github. Among the collected tweets, those that not directly related to the Stratis blockchain platform were removed. As a result, we found 5,085 tweets with the information of time/date posted, number of retweets, and number of favorites. We further divided the collected tweets by the period of posting (i.e., pre-ICO period, ICO period, post-ICO period) and by the user account group (i.e., Stratis developer vs. general public) to explore the differences in topics. Table 1 presents the overview of the collected tweet data.

Table 1. Number of tweets by period/user account

\begin{tabular}{|c|c|c|c|c|}
\hline & $\begin{array}{c}\text { Pre-ICO } \\
(5 / 22- \\
6 / 20)\end{array}$ & $\begin{array}{c}\text { ICO } \\
(6 / 21- \\
7 / 26)\end{array}$ & $\begin{array}{c}\text { Post-ICO } \\
(7 / 27- \\
8 / 26)\end{array}$ & Total \\
\hline Stratis & 4 & 78 & 26 & 108 \\
\hline $\begin{array}{c}\text { General } \\
\text { Public }\end{array}$ & 627 & 3527 & 823 & 4977 \\
\hline Total & 631 & 3605 & 849 & 5085 \\
\hline
\end{tabular}

\subsection{Data analysis}

With the collected tweet data, we tried to find the most frequently mentioned/retweeted topics from the period/user group. Since most of the major information regarding the blockchain solution is posted by the coin developer and spread throughout the general public, we 
focused on the tweets posted that were frequently retweeted by the Stratis blockchain developer and by the general public. Since little previous research has been done applicable to the cryptocurrency ICO practice, we adopted a qualitatively grounded theory method to identify the topic of each tweet. Methodological grounded theory is widely used in IS studies, developed through an iterative, intensive, data-driven, analysis process of extracting the semantic meaning in the discourses [3].

For the research purpose, we filtered the data so that only tweets that were frequently $r e$-tweeted were considered for the analysis. After the filtering, two coders read the tweets and reviewed the iterative coding process suggested by Corbin and Strauss [3], which includes open coding, axial coding, and selective coding to find the topic of each tweet posted. After the initial coding, the inter-coder agreement rate was $91.5 \%$. Next, the coders reviewed the resolution process and agreed with the reconciled coding outcome for the topic of each tweet.

\section{Results}

From the analysis of the data, we found several patterns of tweets regarding the Stratis ICO project that were popularly shared. Table 2 presents the topics captured from the tweets posted by the Stratis platform developer. First, in pre-ICO period, tweets advertising both the company and the ICO posted by the coin developer are mostly retweeted. Additionally, the developer's tweets for sharing information of the cryptocurrency were also popularly retweeted. Meanwhile, during the ICO period, more diverse topics appeared to be posted and shared in the developer's tweets. The most frequently retweeted topic in the period was about the ICO event, which announced that the ICO was occurring. Additional information sharing regarding the technology, the white paper of the ICO project, and the management team was also retweeted. Lastly, updates regarding the status of funding was also one of the frequently shared tweets in the period. After the ICO concluded, more information was shared regarding the company and the cryptocurrency technology. The updates of the funding and trading were also retweeted frequently during this period. It is interesting to see that a scam warning was also shared many times after an ICO project-funding event. We posit that this scam warning stemmed from the recorded high ROI status in the public.

Table 2. Topics of the tweets posted by Stratis

\begin{tabular}{|c|c|c|c|}
\hline Period & Topic & $\begin{array}{c}\text { Number } \\
\text { of } \\
\text { tweets }\end{array}$ & $\begin{array}{l}\text { Total count } \\
\text { of being } \\
\text { retweeted }\end{array}$ \\
\hline \multirow{2}{*}{$\begin{array}{l}\text { Pre- } \\
\text { ICO } \\
\text { period }\end{array}$} & $\begin{array}{l}\text { ICO } \\
\text { Advertisement }\end{array}$ & 2 & 165 \\
\hline & $\begin{array}{l}\text { Technology } \\
\text { Information }\end{array}$ & 2 & 628 \\
\hline \multirow{6}{*}{$\begin{array}{l}\text { ICO } \\
\text { period }\end{array}$} & $\begin{array}{l}\text { ICO } \\
\text { Advertisement }\end{array}$ & 47 & 5621 \\
\hline & $\begin{array}{l}\text { Technology } \\
\text { Information }\end{array}$ & 14 & 2500 \\
\hline & $\begin{array}{l}\text { Advertisement - } \\
\text { Company }\end{array}$ & 6 & 579 \\
\hline & $\begin{array}{l}\text { Developer } \\
\text { Information }\end{array}$ & 7 & 524 \\
\hline & White Paper & 2 & 449 \\
\hline & $\begin{array}{l}\text { News - ICO } \\
\text { Status }\end{array}$ & 1 & 137 \\
\hline \multirow{7}{*}{$\begin{array}{l}\text { Post- } \\
\text { ICO } \\
\text { period }\end{array}$} & News - Coin & 7 & 1706 \\
\hline & $\begin{array}{l}\text { Technology } \\
\text { information }\end{array}$ & 6 & 799 \\
\hline & $\begin{array}{l}\text { Advertisement - } \\
\text { Company }\end{array}$ & 4 & 620 \\
\hline & White paper & 2 & 256 \\
\hline & $\begin{array}{l}\text { News - ICO } \\
\text { Status }\end{array}$ & 2 & 246 \\
\hline & $\begin{array}{l}\text { Developer } \\
\text { information }\end{array}$ & 1 & 160 \\
\hline & $\begin{array}{l}\text { News - Scam } \\
\text { warning }\end{array}$ & 1 & 81 \\
\hline
\end{tabular}

Table 3 presents the topics captured from the tweets posted by the public. First, in pre-ICO period, similar to the result of developer's tweets, advertisements of ICO and the developing company are most popular. However, it is clear that people interested in a project also created notices of promotional events and shared personal expectations, which are noteworthy. Second, during the ICO period, information regarding the cryptocurrency technology was retweeted popularly, followed by the ICO news as well as the company/ICO advertisement. Although the project was running, ICO funding status and manager of Stratis information were also shared. 
Last, after the ICO, technology information is the most popular tweets shared, among the people followed by funding status and advertisement of the company. Comparing to Stratis platform generated tweets, the general public created and shared its own information as well. For example, other coin news, personal investment/evaluation of coins, and coin trading information were included during the post-ICO period. More detailed topics are listed in the table 3.

Table 3. Topics of the tweets posted by general public

\begin{tabular}{|c|c|c|c|}
\hline Period & Topic & $\begin{array}{l}\text { Number } \\
\text { of tweets }\end{array}$ & $\begin{array}{l}\text { Total count } \\
\text { of being } \\
\text { retweeted }\end{array}$ \\
\hline \multirow{7}{*}{$\begin{array}{l}\text { Pre- } \\
\text { ICO } \\
\text { period }\end{array}$} & $\begin{array}{l}\text { ICO } \\
\text { advertisement }\end{array}$ & 39 & 755 \\
\hline & $\begin{array}{l}\text { New } \\
\text { Technology } \\
\text { announcement }\end{array}$ & 1 & 544 \\
\hline & $\begin{array}{l}\text { Company } \\
\text { advertisement }\end{array}$ & 4 & 487 \\
\hline & $\begin{array}{l}\text { Technology } \\
\text { information }\end{array}$ & 14 & 135 \\
\hline & $\begin{array}{l}\text { Notice of } \\
\text { promotional } \\
\text { event }\end{array}$ & 14 & 25 \\
\hline & $\begin{array}{l}\text { Information } \\
\text { sharing }\end{array}$ & 1 & 1 \\
\hline & $\begin{array}{l}\text { Personal } \\
\text { Expectation }\end{array}$ & 1 & 1 \\
\hline \multirow{8}{*}{$\begin{array}{l}\text { ICO } \\
\text { period }\end{array}$} & $\begin{array}{l}\text { Technology } \\
\text { Information }\end{array}$ & 62 & 901 \\
\hline & ICO Event & 17 & 877 \\
\hline & $\begin{array}{l}\text { Company } \\
\text { advertisement }\end{array}$ & 9 & 406 \\
\hline & $\begin{array}{l}\text { ICO News - } \\
\text { Funding Status }\end{array}$ & 31 & 398 \\
\hline & $\begin{array}{l}\text { ICO } \\
\text { Advertisement }\end{array}$ & 24 & 373 \\
\hline & $\begin{array}{l}\text { Manager } \\
\text { Information }\end{array}$ & 2 & 155 \\
\hline & $\begin{array}{l}\text { Developer } \\
\text { Information }\end{array}$ & 1 & 33 \\
\hline & $\begin{array}{l}\text { Information } \\
\text { Sharing }\end{array}$ & 2 & 8 \\
\hline \multirow{2}{*}{$\begin{array}{l}\text { Post- } \\
\text { ICO } \\
\text { period }\end{array}$} & $\begin{array}{l}\text { Technology } \\
\text { information }\end{array}$ & 32 & 375 \\
\hline & $\begin{array}{l}\text { ICO News - } \\
\text { Funding Update }\end{array}$ & 3 & 314 \\
\hline
\end{tabular}

\begin{tabular}{|l|l|c|c|}
\hline & $\begin{array}{l}\text { Company } \\
\text { advertisement }\end{array}$ & 23 & 215 \\
\cline { 2 - 4 } & Coin news & 66 & 202 \\
\cline { 2 - 4 } & $\begin{array}{l}\text { Technology } \\
\text { news }\end{array}$ & 1 & 104 \\
\cline { 2 - 4 } $\begin{array}{l}\text { ICO } \\
\text { advertisement }\end{array}$ & 1 & 18 \\
\cline { 2 - 4 } Scam warning & 5 & 11 \\
\hline $\begin{array}{l}\text { Personal } \\
\text { investment }\end{array}$ & 1 & 11 \\
\hline $\begin{array}{l}\text { ICO News } \\
\text { Coin market } \\
\text { request }\end{array}$ & 2 & 6 \\
\cline { 2 - 4 } & $\begin{array}{l}\text { Personal } \\
\text { evaluation }\end{array}$ & 3 & 5 \\
\cline { 2 - 4 } & $\begin{array}{l}\text { Personal coin } \\
\text { market news }\end{array}$ & 4 & 4 \\
\hline & $\begin{array}{l}\text { Coin trading } \\
\text { information }\end{array}$ & 2 & 2 \\
\hline
\end{tabular}

\section{Discussion and Implications}

Following the data analysis, our findings show that each ICO stage reveals both common and distinct information created by either Stratis or the general public's Twitter users. First, given agency theory, our findings indicate that public users generate additional information beyond the Stratis original post. For instance, during the pre-ICO stage, public users created five more additional information to fulfill information asymmetry between Stratis and public users. This trend remains consistent during and following ICO. One interesting fact is that public users play two roles, becoming principal as an information recipient and an agent as an information creator in our research context. Particularly, during ICO stage, public users shared manager information with others and personal evaluation of investment, ICO fund status were added up onto the Stratis publicly shared information. We argue that all of those activities account for the effort of reduction of agency costs, including monitoring, bonding, and residual cost under agency theory. Since prospective investors and people who are interested in earning cryptocurrency suffer from a relative lack of information, they might collectively gather any useful pieces of messages and share them for their peace of mind.

Second, applying signaling theory, in terms of signal credibility, both ICO advertisement and technology 
information were spread the most before and during ICO event, showing that the credibility of ICO project is highly influenced by technology information to minimize risk. During the ICO event, funding status shared widely among the public indicates that it is an important signaling factor for people who are interested in an ICO project. One interesting of finding is that, while manager information generated by Stratis was not detected as being significant, public Twitter users retweeted Stratis manager information or related news many times. Thus, manager information also is counted as a credible signaling factor impacting peoples' interest. Overall, our findings show that, while Stratis propagates multiple signaling cues to public, public Twitter users also become content creators given Stratis's basic information. For example, during the pre-ICO period, public users' own shared information expands the signal spectrum significantly. This trend is obvious in the postICO stage, such that public users added their own experience with other users on top of Statis's official announcement regarding funding status, ICO news, or developer information. Interestingly, public users were aware of the threat by phishing scams via the email. In terms of the number of signals targeting information recipients, there were more informational cues during pre-ICO and post-ICO stages generated by public users than during the ICO stage. This finding shows that public users were actively involved in both pre- and post-ICO stages; thus, user-generated signals were more prevalent than those by Stratis.

Pre-ICO

In-ICO

Post-ICO

\section{Signals by Agent 1 (Information propagation)}

\begin{tabular}{|c|}
\hline Agent 1 \\
Information \\
Provider \\
(Stratis) \\
\\
\hline Information \\
Provider \\
(Public User) \\
Agent 2 \\
\hline
\end{tabular}
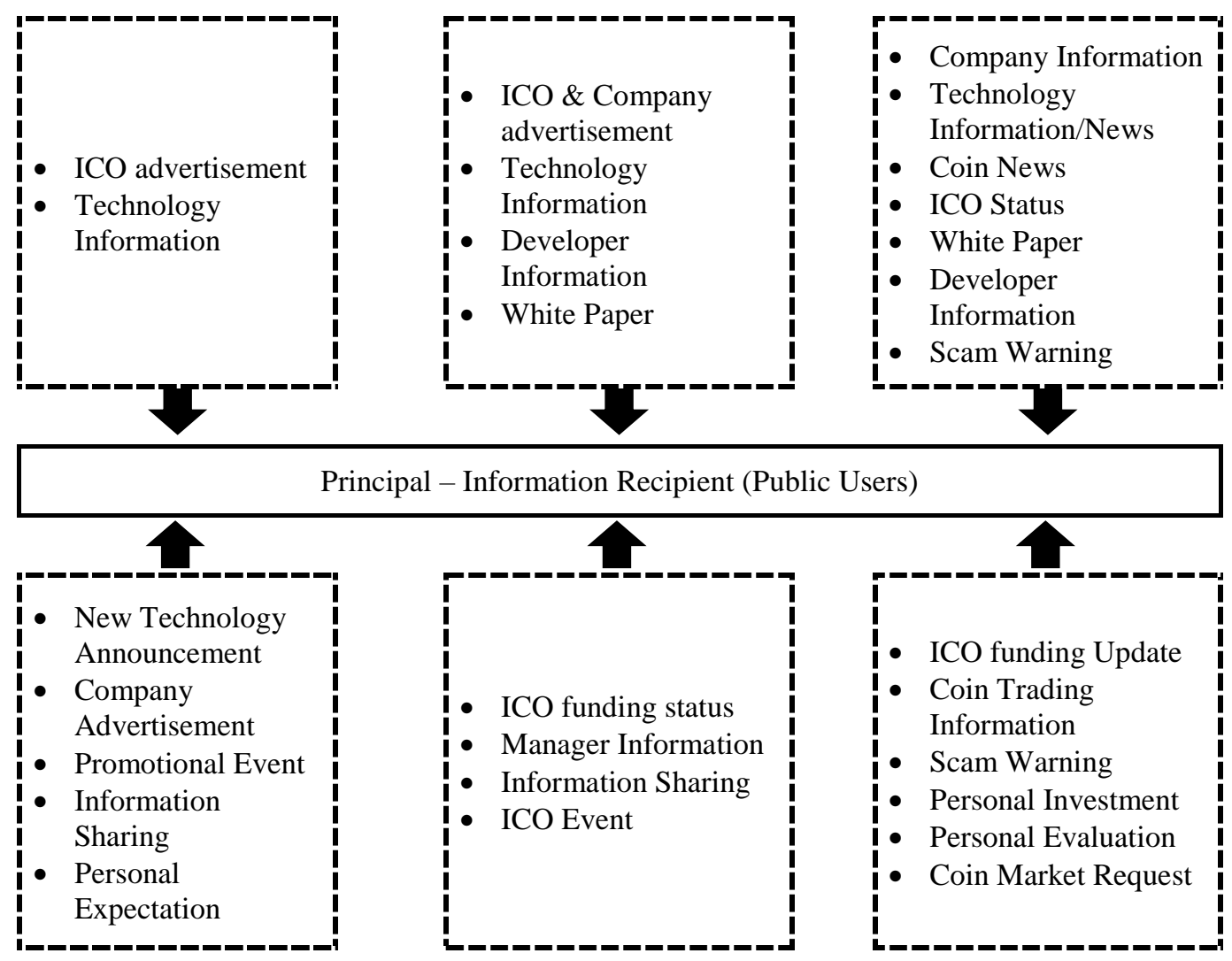

Signals by Agent 2 (Information Propagation)

Figure 1. Flow of informational cues and messages 


\section{Limitations and future research}

Our research is limited following in the following two ways. First, given the research scope, we considered only one ICO project that has achieved high return on investment (ROI) at the time of data collection. However, it would be worthwhile for future investigators to include various ICO projects, such as the least ROI recorded ICO project, the most funded ICO project, the least funded ICO project, and so on. Analyzing different categories of fund amount and ROI, different aspects of signals and information can be found.

Second, because of limited analyzing resources, we only examined tweets that were re-tweeted at least once to other Twitter users to see the information flow. During the data analysis stage, we noticed that some tweets were marked as a favorite, meaning that such information was attractive to the information recipient. Thus, for future research, we suggest noting the combined data that is re-tweeted and marked favorite simultaneously, then compare them to independently retweeted without being marked as a favorite to see if any significant discrepancies emerge in terms of information flow.

\section{Conclusion}

The goal of the current research was to examine what informational messages are generated by an entity of ICO project and public potential investors or those who are interested in ICO project. To achieve our research goal, we employed agency and signaling theories and extracted tweets related to the Stratis ICO project before, during and after ICO project. Findings revealed that a variety of ICO related messages were created by both Stratis and public Twitter users interested in the ICO project. Particularly, public Twitter users added their own informational messages and shared them with others through Twitter channels. Among many topics, technology related information, ICO news and advertisements, funding status and manager information were among the most frequently shared information messages. Our research argues that Stratis and public users' messages fulfill agency cost under agency theory and messages themselves play an important role in signaling effect on the success of ICO, since Stratis is achieving the highest ROI among ICO projects at the time of study.

\section{References}

[1] www.coinmarketcap.com, accessed April 25, 2018.

[2] W. Boulding, and A. Kirmani, "A Consumer-Side Experimental Examination of Signaling Theory: Do Consumers Perceive Warranties as Signals of Quality?", Journal of Consumer Research, 20(1), 1993, pp. 111-123.

[3] J. Corbin, and A. Strauss, Basics of Qualitative Research: Techniques and Procedures for Developing Grounded Theory, Thousand Oaks, CA: Sage publications, 2014.

[4] https://www.cryptocompare.com/coins/guides/what-isstratis/, accessed May 2018.

[5] K. M. Eisenhardt, "Agency Theory: An Assessment and Review", Academy of Management Review, 1989, pp. 57-74.

[6] V. Gurbaxani, and S. Whang, "The Impact of Information Systems on Organizations and Markets", Communications of the ACM, 34(1), 1991, pp. 59-73.

[7] C. Harwick, "Cryptocurrency and the Problem of Intermediation", The Independent Review, 20(4), 2016, pp. 569-588.

[8] D. Jun, and M. A. Vasarhelyi, "Toward Blockchain-Based Accounting and Assurance", Journal of Information Systems, 31(3), 2017, pp. 5-21.

[9] M. Keil, H. J. Smith, S. Pawlowski, and L. Jin, "'Why Didn't Somebody Tell Me?': Climate, Information Asymmetry, and Bad News About Troubled Projects", ACM Sigmis Database, 35(2), 2004, pp. 65-84.

[10] A. Kirmani, and A. R. Rao, "No Pain, No Gain: A Critical Review of the Literature on Signaling Unobservable Product Quality", Journal of Marketing, 64(2), 2000, pp. 66-79.

[11] N. Kshetri, "Blockchain's Roles in Strengthening Cybersecurity and Protecting Privacy", Telecommunications Policy, 41(10), 2017, pp. 1027-1038.

[12] R. Martenson, "Corporate Brand Image, Satisfaction and Store Loyalty: A Study of the Store as a Brand, Store Brands and Manufacturer Brands", International Journal of Retail \& Distribution Management, 35(7), 2007, pp. 544-555.

[13] S. Nakamoto, "Bitcoin: A Peer-to-Peer Electronic Cash System", 2008, 
[14] P. A. Pavlou, H. Liang, and Y. Xue, "Understanding and Mitigating Uncertainty in Online Exchange Relationships: A Principal - Agent Perspective", MIS Quarterly, 31(1), 2007, pp. 105-136.

[15] G. W. Peters, and E. Panayi, "Understanding Modern Banking Ledgers through Blockchain Technologies: Future of Transaction Processing and Smart Contracts on the Internet of Money": Banking Beyond Banks and Money, Springer, 2016, pp. 239-278.

[16] A. R. Rao, Q. Lu, and R. W. Ruekert, "Signaling Unobservable Product Quality through a Brand Ally", Journal of Marketing Research (JMR), 36(2), 1999, pp. 258-268.

[17] K. A. Sontakke, and A. Ghaisas, "Cryptocurrencies: A Developing Asset Class", International Journal of Business Insights \& Transformation, 10(2), 2017, pp. 10-17.

[18] M. Spence, "Job Market Signaling", Quarterly Journal of Economics, 87(3), 1973, pp. 355-374.

[19] https://steemit.com/crypto-news/@ byron-hazard/stratisico-summary-latest-update-stratisplatform, accessed May, 2018.

[20] D. Tapscott, and A. Tapscott, Blockchain Revolution: How the Technology Behind Bitcoin Is Changing Money, Business, and the World, Penguin, 2016.

[21] C. Trew, G. Brandon, and N. Dorier, "Stratis Whitepaper: The Vision and the Possibilities", in (Editor, 'ed.'^'eds.'): Book Stratis Whitepaper: The Vision and the Possibilities, Stratisplatform, 2016

[22] J. D. Wells, J. S. Valacich, and T. J. Hess, "What Signal Are You Sending? How Website Quality Influences Perceptions of Product Quality and Purchase Intentions", MIS Quarterly, 35(2), 2011, pp. 373-A318. 